\title{
Generation of Coherent, Broadband X-Ray and Mid-IR Pulses in a Noble-Gas-Filled Hollow Waveguide
}

\author{
Jeremy Pigeon, Sergei Tochitsky and Chan Joshi \\ Neptune Laboratory, Department of Electrical Engineering, University of California, Los Angeles, California, \\ 90095

\begin{abstract}
We discuss a proposed experiment on guiding of a high-repetition rate, $20 \mathrm{GW}, \mathrm{CO}_{2}$ laser beam in a gas-filled hollow glass waveguide. Extended interaction of the 3 ps pulses with a gas Kerr medium will be used for measurements of nonlinearity at $10 \mathrm{um}$, and supercontinuum generation followed by pulse compression. Feasibility of 3-5 keV X-ray production via $\mathrm{HHG}$ is presented.
\end{abstract}

Keywords: $\mathrm{CO}_{2}$ laser, waveguide, nonlinear index, self-phase modulation, pulse compression PACS: 42.55.Lt , 42.65.Jx ,42.65.Re

\section{INTRODUCTION}

Recent work has demonstrated possible advantages of using a high power, mid-IR laser in studying the acceleration of ions [1] and discussed X-ray generation via HHG [2]. The main advantage of using a $\mathrm{CO}_{2}$ laser can be attributed to the 10 um wavelength of the laser light. In the case of ion acceleration this long wavelength regime provides an opportunity to study over-dense laser plasma interactions in a gas jet or cell [1]. In the case of HHG, the 10 um wavelength could open an avenue to obtain 3-5 keV X-rays due to the quadratic scaling of the HHG cut-off with wavelength [2]. The $\mathrm{CO}_{2}$ gain medium has a high damage threshold, determined by the ionization of the active medium on the order $10^{12} \mathrm{~W} / \mathrm{cm}^{2}$, which removes the necessity of pulse stretching so that direct amplification to very high peak power can be realized. However, amplification of picosecond pulses in a $\mathrm{CO}_{2}$ gas medium is hindered by a relatively narrow bandwidth. A multi-atmosphere laser is suitable for picosecond pulse amplification but these devices often have a small discharge volume which limits the maximum pulse energy.

Recently 15 TW of peak power was demonstrated using the UCLA Neptune Laboratory's master oscillator power-amplifier (MOPA) chain [3] which relies on a multi-atmosphere final amplifier that can deliver a shot once every five minutes. However, there are many applications that would benefit from a system that operates at a high repetition rate, for which only atmospheric $\mathrm{CO}_{2}$ amplifiers (TEA systems) are readily available. Here we first describe the development of a $1 \mathrm{~Hz}$ repetition rate, $20 \mathrm{GW}, 3$ ps $\mathrm{CO}_{2}$ laser based on final amplification at $1 \mathrm{~atm}$. Second, we propose an experiment to guide our high repetition rate laser in a commercially available, Ag/AgI coated hollow glass waveguide (HGW) for studies in nonlinear optics. The end goals of such an experiment is: first, to measure the Kerr index in noble gases and, second, to increase our peak power to $100 \mathrm{GW}$ by using chirping caused by self-phase modulation and subsequent compression of our laser pulses. Finally we discuss the feasibility of X-ray production via high harmonic generation using a 10 um pump.

\section{AMPLIFICATION OF PICOSECOND PULSES IN $\mathrm{CO}_{2}$ LASERS}

The main technological challenge in high power $\mathrm{CO}_{2}$ laser development is that, at 1 atm of pressure, the $\mathrm{CO}_{2}$ gain medium does not have sufficient bandwidth to support the amplification of picosecond pulses. Figure (1a) is a simulated $\mathrm{CO}_{2}$ gain spectrum at $1 \mathrm{~atm}$ of pressure. At atmospheric pressure the bandwidth of the $10 \mathrm{P}$ branch is comprised of individual $3.5 \mathrm{GHz}$ rovibrational lines separated by $55 \mathrm{GHz}$. Therefore, in order to amplify picosecond pulses in $\mathrm{CO}_{2}$ one must rely on two broadening mechanisms: pressure and field broadening.

\section{Pressure Broadening}

The width of the individual rovibrational lines in the $\mathrm{CO}_{2}$ gain medium is $\Delta v \approx 3.5 \mathrm{GHz} / \mathrm{atm}$. At sufficiently high pressure, the mean free time in the gain medium is faster than the transition time for stimulated emission which 
results in collisional broadening of the rovibrational lines. Figure (1b) is a simulated $\mathrm{CO}_{2}$ gain spectrum at 10 atm, here the rovibrational lines have been broadened to $\sim 35 \mathrm{GHz}$ providing a quasi-continuum over approximately 1.2 THz. The broadening in a laser gas mix consisting of $\mathrm{CO}_{2}, \mathrm{He}$ and $\mathrm{N}_{2}$ can be approximated as [4],

$$
\Delta v_{\text {pressure }}=P\left(5.79 \Psi_{\mathrm{CO}_{2}}+4.25 \Psi_{N_{2}}+3.55 \Psi_{\mathrm{He}}\right)
$$

Where, in equation (1), $\Psi_{S}$ is the partial pressure of the pertinent gas species. It should be noted that, at 25 atm, the gain medium loses its modulation and becomes a true continuum across $1.2 \mathrm{THz}$ of bandwidth over the entire vibrational branch. Unfortunately it is technologically impractical to design a large aperture, discharge pumped amplifier which operates at greater than $10 \mathrm{~atm}$ of pressure [5].

\section{Field Broadening}

Field broadening arises when the amplified pulse is intense enough to perturb the energy levels of the quantum system resulting in a smearing of these levels, similar to the AC Stark Effect [6]. The bandwidth gained through field broadening can be expressed as [7],

$$
\Delta v_{\text {field }} \approx \Omega_{R}=1.38 \cdot 10^{7} \mu \sqrt{I}
$$

In the above, $\Omega_{R}$ is the Rabi frequency, $\mu$ is the $\mathrm{CO}_{2}$ transition dipole moment in Debye, and I is the laser intensity in $W / \mathrm{cm}^{2}$. For the $10.6 \mathrm{um}$ lasing transition, the dipole moment is approximately 0.0275 D [8]. The total broadened line width is then the sum of the contribution from pressure and field broadening,

$$
\Delta v_{\text {Total }}=\Delta v_{\text {Pressure }}+\Delta v_{\text {Field }}
$$

At a seed intensity of $5 \mathrm{GW} / \mathrm{cm}^{2}$ a 1 atm amplifier's gain spectrum becomes very similar to the 10 atm spectrum of figure (1b). Therefore, using a sufficiently intense seed one should be able to use atmospheric pressure $\mathrm{CO}_{2}$ lasers to amplify picosecond pulses. Such atmospheric devices, $\mathrm{TEA} \mathrm{CO}_{2}$ lasers, are commercially available and can operate at a pulse repetition frequency of $1-100 \mathrm{~Hz}$ and an output energy of 1-100J.

Due to residual modulation in the gain spectrum, amplification of picosecond pulses at 10 atm results in a pulse train in the time domain. This pulse train contains 3 ps long pulses separated by $18 \mathrm{ps,} \mathrm{a} \mathrm{time} \mathrm{constant} \mathrm{related} \mathrm{to} \mathrm{the}$ $1 / 55 \mathrm{GHz}$ modulation in the frequency domain. Figure (1c) depicts simulation results of a typical output pulse train, generated by seeding a $10 \mathrm{~atm}$ device with a single picosecond pulse.
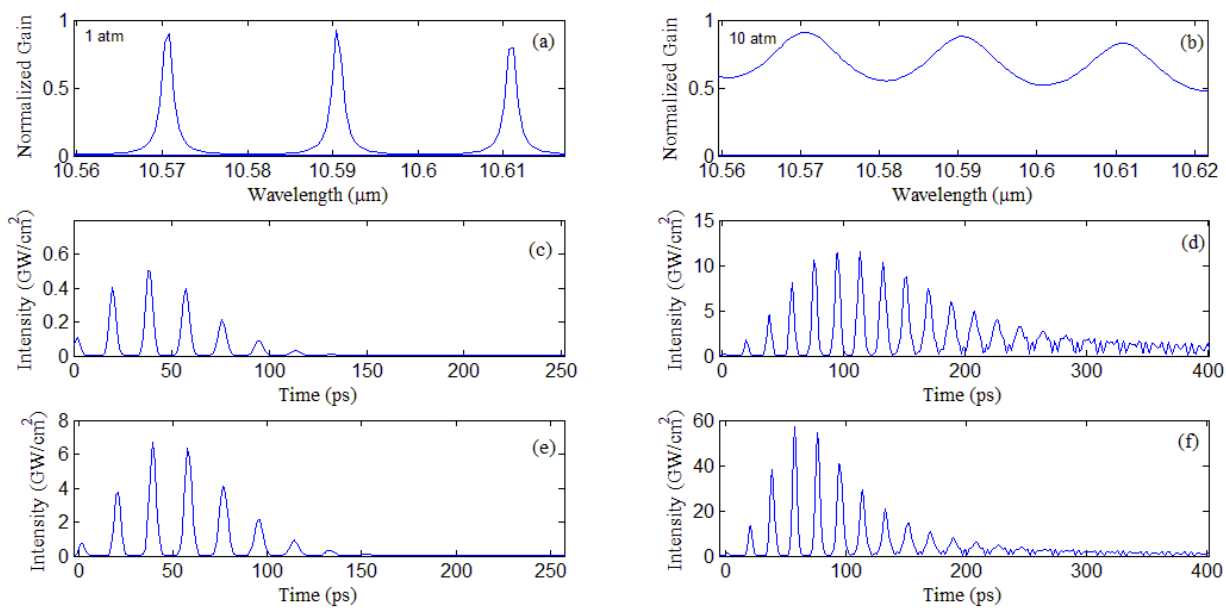

FIGURE 1. (a) Simulated $\mathrm{CO}_{2}$ gain spectrum in the vicinity of the $10 \mathrm{P}(20)$ line at $1 \mathrm{~atm}$ and (b) at 10 atm of pressure. Temporal profiles of $\mathrm{CO}_{2}$ laser pulses at $0.5 \mathrm{GW} / \mathrm{cm}^{2}$ seeded into a $1 \mathrm{~atm}$ module (c) and corresponding output after $3 \mathrm{~m}$ of amplification (d). Temporal profiles of $\mathrm{CO}_{2}$ laser pulses at $6 \mathrm{GW} / \mathrm{cm}^{2}$ seeded into a $1 \mathrm{~atm}$ module (e) and corresponding output after $3 \mathrm{~m}$ of amplification (f). 
Figures (1c-1f) summarize results of simulations showing the effect of field broadening in a $1 \mathrm{~atm}$ device. Figure (1c) and (1d) are the respective input and output pulse trains for a case when the input intensity is approximately $0.5 \mathrm{GW} / \mathrm{cm}^{2}$. Figure (1e) and figure (1f) are the same simulation with the seed intensity scaled to $6 \mathrm{GW} / \mathrm{cm}^{2}$. Due to the intense field depicted in figure (1e), the 3 ps pulse train of figure (1f) was amplified with negligible broadening.

\section{NEPTUNE LABORATORY'S 20 GW, 3 PS, HIGH REPETITION RATE $\mathrm{CO}_{2}$ LASER SYSTEM}

We have recently developed a high repetition rate laser system which relies on a 1 atm device for final amplification. The system delivers $20 \mathrm{GW}, 3 \mathrm{ps}, 10 \mathrm{um}$ pulses operating at a repetition frequency of $1 \mathrm{~Hz}$ [9]. The laser system consists of MOPA stages with two amplification regimes. In the first regime the pulse is amplified from $\mathrm{nJ}$ to $\mathrm{mJ}$ levels in $8 \mathrm{~atm} \mathrm{CO}_{2}$ amplifiers. In the final stage the pulse is amplified to Joule energies in a $1 \mathrm{~atm}$ booster amplifier where the laser field provides the bandwidth to sustain the pulse duration [9].

Figure (2) shows a typical pulse train after the final stage of amplification, as measured by a streak camera. Similar to modeling, the pulse train consists of $\sim 3$ ps pulses separated by 18 ps. Specific details regarding the system and the measurement presented in figure (2) are discussed elsewhere [9].

These results show that 3 ps pulses can be amplified in an atmospheric $\mathrm{CO}_{2}$ laser, for which the bandwidth is predominantly provided by field broadening. Such a system, operating at a high repetition rate, lends itself to studies which are difficult or impossible to perform in a single shot experiment.
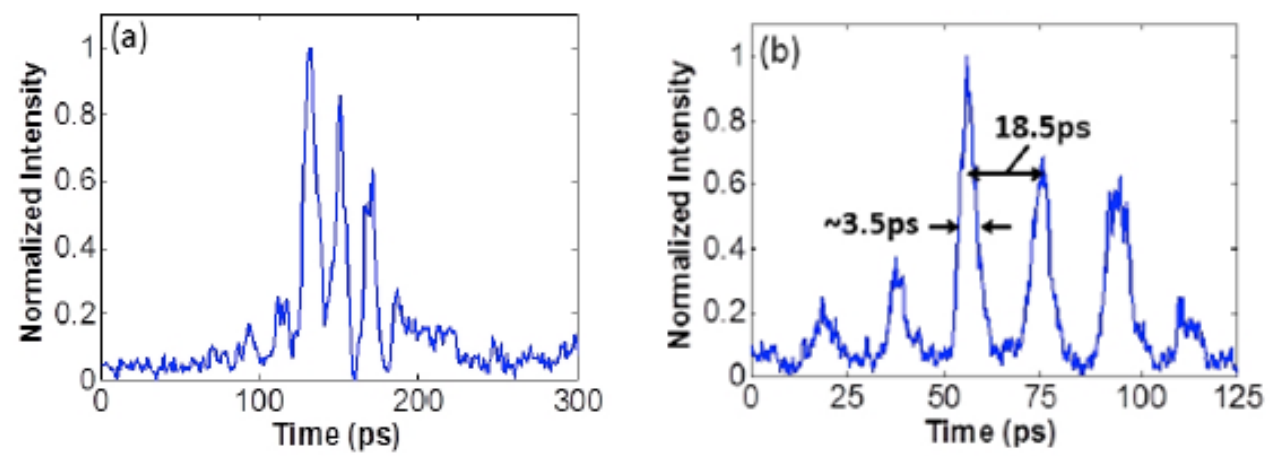

FIGURE 2. (a) A typical pulse train as measured by a streak camera and (b) zoomed image of the same data in (a) depicting a 3 ps pulse train separated by $18 \mathrm{ps}$.

\section{NONLINEAR OPTICS IN A NOBLE GAS-FILLED HOLLOW GLASS WAVEGUIDE}

As of late there has been a tremendous interest in ultrafast nonlinear optics, particularly in studies of broadband (supercontinuum) radiation generation in gas-filled hollow core fibers [14], laser filamentation in the air [21] and Xray production via HHG [20]. The vast majority of these studies have been performed in the near-IR ( $0.8-1 \mathrm{um})$. Despite the apparent interest in extending these studies to longer wavelengths, there have been no such experiments in the mid-IR except one very recent report on using a 0.8 um pumped OPA at 3.9 um for nonlinear optics studies [19].

\section{Efficient Guiding of 10 um Light in a HGW}

Commercially available, $\mathrm{Ag} / \mathrm{AgI}$ coated hollow glass waveguides [23] have been explored as a method to guide high-power, short pulses at $10 \mathrm{um}$, the results of which have demonstrated $98 \%$ efficient guiding over $30 \mathrm{~cm}$ in air [10]. These results were obtained when the laser was properly matched to the $\mathrm{EH}_{11}$ mode of the waveguide for which the matching condition is $\frac{w_{0}}{a}=0.64$. Here $a$ is the radius of the waveguide and $w_{0}$ is the beam radius [11]. 
The first advantage of using a waveguide in studying nonlinear optics can be attributed to the fact that guiding the light removes uncertainty regarding the laser's intensity distribution and extends the interaction length well beyond that limited by diffraction. A second critical advantage of using a waveguide is that, when coupled to the $\mathrm{EH}_{11}$ mode, the laser maintains a linear polarization which is perpendicular to the axis of the guide [11]. This polarization preservation is crucial for the proposed experiment explained in the next section.

\section{Proposed Measurement of Kerr Indices using a HGW}

The standard way for determining the nonlinear index of refraction is measuring third/fifth harmonic yield in the medium of interest $[12,19]$. However, due to the complicated temporal structure of the $\mathrm{CO}_{2}$ pulse train, a simple measurement of harmonic yield is ambiguous. In order to measure the nonlinear index of refraction in noble gases, we propose to use a method of polarization rotation, namely to use a gas-filled HGW as a Kerr cell placed between two crossed polarizers. In this scheme, depicted in figure (3), the 10 um laser acts to rotate the polarization of a 630 $\mathrm{nm}$ probe beam. Upon rotation, the $630 \mathrm{~nm}$ probe is leaking through an analyzer, originally set to minimize the transmission of the probe, and this signal is sent to a streak camera to measure the temporal structure of the probe. It is important to note that the temporal structure carried by the 10 um pump pulse is transcribed unto the probe signal through this process.

After the probe's temporal distribution is measured by the streak camera, we now have several pulses of different intensity which can be individually fit to Malus's law:

$$
T(t)=A \sin ^{2}\left[2.34 \cdot 10^{6}\left(\frac{\pi L}{\lambda}\right) n_{2} I(t)\right]
$$

In the above, $\mathrm{A}$ is the attenuation of the probe beam, $\mathrm{L}$ is the length of the waveguide, $n_{2}$ is the nonlinear index of refraction in $\mathrm{cm}^{2} / W$ and $\mathrm{I}$ is the laser intensity in $W / \mathrm{cm}^{2}$ [13]. On each shot we will measure the transmission of the probe as a function of pump intensity and extract $n_{2}$ as a fitting parameter. Preliminary analysis show that a 5\% change in the probe's transmission results in a $\sim 7 \%$ change in $n_{2}$. It should be mentioned that recently measured values of $n_{2}$ at 0.8 um are quoted with uncertainties on the order of $10 \%$ [22]. After averaging the nonlinear index over many shots, we will fit the index as a function of pressure from 100-300 torr, noting that the nonlinear index should vary linearly with pressure.

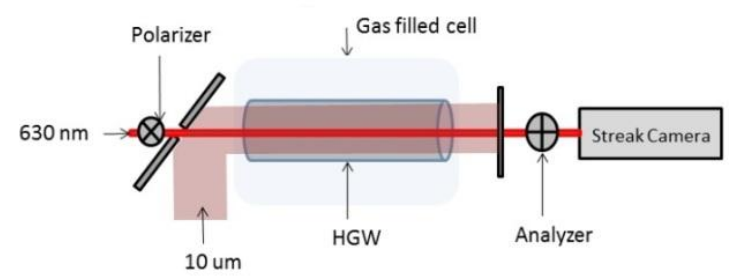

FIGURE 3. Schematic for a proposed experiment to measure the nonlinear index of refraction.

In order to ensure that we measure the transient birefringence related only to the nonlinear index of refraction we must operate at a plasma free condition. We will perform these experiments in the vicinity of 100 torr to ascertain that we are free of avalanche ionization and we will ensure that our pump power remains well below the critical power of self-focusing yet guide the beam using the HGW. It should be noted that this measurement can be easily extended to very high intensities on the order of $10^{12}-10^{13} \mathrm{~W} / \mathrm{cm}^{2}$ where a possible saturation of $n_{2}$ may be detected [22].

\section{PROPOSED $100 \mathrm{GW}$, HIGH REPETITION RATE $\mathrm{CO}_{2}$ LASER SYSTEM BASED ON CHIRPING AND COMPRESSION}

With knowledge of the nonlinear index of refraction for noble gases at 10 um we plan to study spectral broadening from self-phase modulation in a HGW. The goal of such a study is to chirp and compress our picosecond pulses in order to increase their peak power. This technique is often used at 1 um in order to produce pulses shorter 
than those limited by the gain bandwidth [14] but has never been demonstrated in the mid-IR. In the picosecond regime one may approximate the increase in the pulse's time bandwidth product by [15],

$$
\Delta f \Delta \tau \approx \sqrt{1+\left(\frac{2 \pi n_{2} I_{0} L}{\lambda}\right)^{2}}
$$

For $I_{0}=6 \mathrm{TW} / \mathrm{cm}^{2}, n_{2 X e}=8 \cdot 10^{-19} \mathrm{~cm}^{2} / W$ [16] and $L=200 \mathrm{~cm}$ we calculate an increase in bandwidth by a factor of 5 for an initially 3 ps, 10 um pulse. Figure (4a) is a plot of the input and output pulse spectrum after 2 meters of propagation in Xenon. It is important to note that propagation through noble gases, like most materials, will place a positive linear chirp on the pulse virtue of the gas's positive group velocity dispersion (GVD) in the spectral range of interest [15]. Although the calculation above was performed as an illustrative example, recent numerical simulations using the generalized nonlinear Schrödinger equation have found that the broadening induced by self-phase modulation in noble gases can be substantial in the mid-IR [16].

If the chirp induced on the pulse is linear then one could envision a scheme to compress our pulses by passing the radiation through a material with a negative GVD. An example of such a material in the mid-IR is $\mathrm{NaCl}$; the insert of figure (1a) is a plot of the group velocity versus wavelength for $\mathrm{NaCl}$ which shows the negative GVD of the material. If we further assume that the level of spectral broadening is on the order of a factor of five then we should be able to increase our peak power from its current level of $20 \mathrm{GW}$ to $\sim 100 \mathrm{GW}$. One critical feature of this scheme is that both the $\mathrm{HGW}$ and the $\mathrm{NaCl}$ plate have very high transmissions, $98 \%$ and $92 \%$, respectively [10]. Figure (4b) is a block diagram of this set-up.

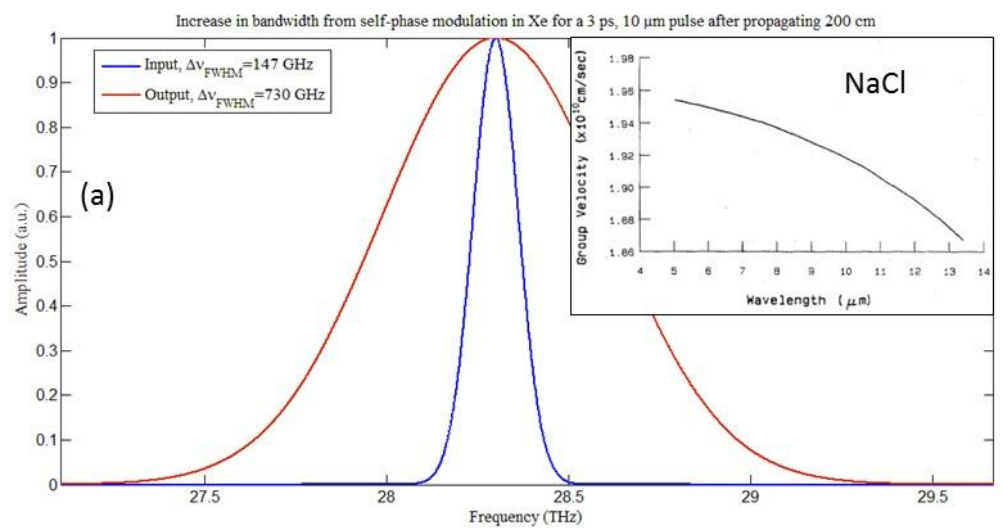

(b)

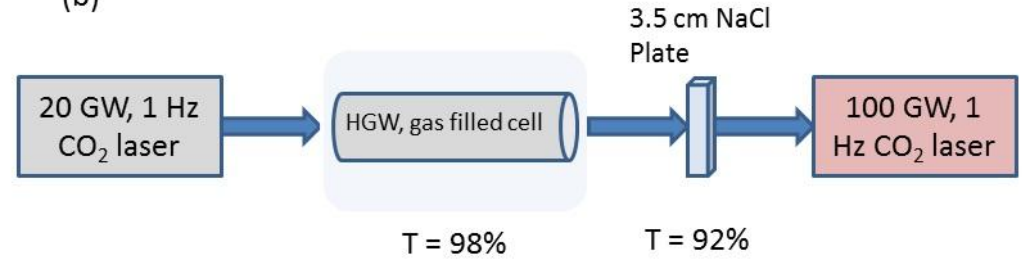

FIGURE 4. (a) Input and output spectral profile for a 3 ps pulse after self-phase modulation in $200 \mathrm{~cm}$ of Xenon gas, insert is the GVD of $\mathrm{NaCl}$ (data taken from [17]) and (b) block diagram of a scheme to increase our peak peak power from $20 \mathrm{GW}$ to 100 GW through chirping and compression.

\section{FEASIBILITY OF X-RAY GENERATION VIA HHG IN THE MID-IR}

The need for X-rays for a myriad of scientific applications has driven research in X-ray sources for decades. One avenue for such a source is high harmonic generation (HHG). The novelty of HHG is that, using a relatively compact, femtosecond laser, one can produce a coherent beam of X-ray radiation [20]. X-ray photons produced via HHG have a characteristic cut-off energy which can be calculated as, 


$$
E_{\max }=I_{p}+3.17 U_{p}
$$

Where $I_{p}$ is the ionization potential of the atom and $U_{p}$ is the ponderomotive energy of the laser given as,

$$
U_{p}=E_{0}^{2} / 4 \omega^{2}
$$

Equations (5) and (6) imply that there is a certain advantage in using long wavelength drive lasers in HHG [2]. With a 10 um pump, intensity of $1-5 \cdot 10^{14} \mathrm{~W} / \mathrm{cm}^{2}$ yield harmonic cut-offs of $3-5 \mathrm{KeV}$, respectively.

Although the scaling of maximum X-ray energy is favorable, numerical simulations have shown that the microscopic efficiency of HHG scales as $\lambda^{-5.5 \pm 0.5}$ [18]. This very rapid drop in microscopic efficiency is attributed to electron wave-packet spreading in the continuum and represents the most significant challenge in using a 10 um laser to produce X-rays via HHG. The microscopic efficiency can be compensated through phase matching between the harmonics and the pump laser, typically performed by increasing the pressure of the medium. However, a recent experimental investigation using a 3.9 um pump laser has shown that, in order to have phase matching, one must produce HHG at $30 \mathrm{~atm}$ of pressure [20]. This finding indicates that phase matching at long wavelengths may prove to be a serious technological challenge.

\section{CONCLUSIONS}

Although high power $\mathrm{CO}_{2}$ lasers provide certain advantages in studies related to advanced acceleration and to radiation generation, present $\mathrm{CO}_{2}$ laser technology is limited by low repetition rate, multi-atmosphere final amplifiers. Here we have shown that nonlinear optical phenomena, mainly field broadening and self-phase modulation, can provide a viable route to extend $0.1-1 \mathrm{TW} \mathrm{CO}_{2}$ lasers to a high repetition rate, all without the need for chirped pulse amplification. We have demonstrated the promise of field broadening through the development of a $1 \mathrm{~Hz}, 20 \mathrm{GW} \mathrm{CO}_{2}$ laser system which relies on an atmospheric final amplifier. The future work will be focused on using self-phase modulation, chirping and compression to increase the peak power of this system at a high repetition rate.

\section{ACKNOWLEDGEMENTS}

This work was supported by U.S. Department of Energy grant DE-FG03-92ER40727.

\section{REFERENCES}

1. D.J. Haberberger, et. al., Nature Phys. 8, 95-99 (2012).

2. T. Popmintchev, et. al., PNAS 106, 10516-10521 (2009).

3. D. Haberberger, S. Tochitsky and C. Joshi, Opt. Express 18(17), 17865-17875 (2010).

4. R.K. Brimacombe and J. Reid, IEEE J. Quant. Elect. 58(3), 1141-1145 (1985).

5. O.R. Wood, Proceedings of the IEEE 62(3), 355-397 (1974).

6. S.H. Autler and C.H. Townes, Phys. Rev. 100(2), 703-722 (1955).

7. R.K. Brimacombe and J. Reid, J. Appl. Phys. 58(3), 1141-1145 (1985).

8. L.S. Rothman, et. al., J. Quant. Spectrosc. Radiat. Transf. 110, 533-572 (2009).

9. S. Ya Tochitsky, J.J. Pigeon, D.J. Haberberger, C. Gong and C. Joshi, Optics Express 20(13), 13762-13768 (2012).

10. C. Sung, S. Ya Tochitsky and C. Joshi, Eleventh AAC Workshop, 512-518 (2004).

11. B. Cros, et. al., IEEE Transactions on Plasma Science 28(4), 1071-1077 (2000).

12. H.J. Lehmeier, W. Leupacher and A. Penzkofer, Opt. Comm. 56(1), 67-72 (1985).

13. T.C. Owen, L.W. Coleman and T.J. Burgess, Appl. Phys. Lett. 22(6), 272-273 (1973).

14. I. Ahmad, et. al., New J. Phys. 13, 093005 (2011).

15. A.E. Siegman, Lasers (University of Science Books, Mill Valley, 1986).

16. V.M. Gordienko, V.T. Platonenko and A.F. Sterzhantov, Quant. Elect. 39(7), 663-668 (2009).

17. P.B. Corkum, IEEE J. Quant. Elect. 21(3), 216-232 (1985).

18. J. Tate, et. al., Phys. Rev. Lett..98(1), 013901 (2007).

19. D. Kartashov, et. al., Opt. Lett. 37(12), 2268-2270 (2012).

20. T. Popmintchev, et. al., Science 336, 1287-1291 (2012).

21. L. Berge, S. Skupin, R. Nuter, J. Kasparian and J.P. Wolf, Rep. Prog. Phys. 70, 1633-1713 (2007).

22. V. Loriot, E. Hertz, O. Faucher and B. Lavorel, Opt. Exp. 17(16), 13429-13434 (2009).

23. R.K. Nubling and J.A. Harrington, Appl. Optics 34(3). 372-380 (1996). 